\title{
The well-posedness of an anisotropic parabolic equation based on the partial boundary value condition
}

Huashui Zhan*

\section{"Correspondence:}

huashuizhan@163.com School of Applied Mathematics, Xiamen University of Technology, Xiamen, 361024, P.R. China

\section{Abstract}

Consider the anisotropic parabolic equation with the variable exponent

$$
u_{t}=\sum_{i=1}^{N}\left(a_{i}(x)\left|u_{x_{i}}\right|^{p_{i}(x)-2} u_{x_{i}}\right)_{x_{i}}
$$

with $a_{i}(x), p_{i}(x) \in C^{1}(\bar{\Omega}), p_{i}(x)>1, a_{i}(x) \geq 0$. If some of $\left\{a_{i}(x)\right\}$ are degenerate on the boundary, a partial boundary value condition is imposed, the stability of weak solutions can be proved based on the partial boundary value condition.

MSC: 35K55; 35K92; 35K85; 35R35

Keywords: anisotropic parabolic equation; partial boundary value condition; the well-posedness

\section{Introduction}

The equation

$$
u_{t}=\operatorname{div}\left(a(x)|\nabla u|^{p(x)-2} \nabla u\right), \quad(x, t) \in Q_{T},
$$

comes from the so-called electrorheological fluids theory (see $[1,2]$ ), where $\Omega \subset \mathbb{R}^{N}$ is a bounded domain with smooth boundary $\partial \Omega, p(x)>1$ is a measurable function. If $a(x) \equiv 1$, there are many related papers to study equation (1.1) with the usual initial-boundary value conditions

$$
\begin{aligned}
& u(x, 0)=u_{0}(x), \quad x \in \Omega, \\
& u(x, t)=0, \quad(x, t) \in \partial \Omega \times(0, T),
\end{aligned}
$$

one can see [3-5] and the references therein.

If $a(x)>0$ when $x \in \Omega$ but $\left.a(x)\right|_{x \in \partial \Omega}=0$, then the stability of weak solutions can be proved without the boundary value condition (1.3), provided that the diffusion coefficient $a(x)$ satisfies some other restrictions. One can see our previous works [6-8].

(c) The Author(s) 2017. This article is distributed under the terms of the Creative Commons Attribution 4.0 International License (http://creativecommons.org/licenses/by/4.0/), which permits unrestricted use, distribution, and reproduction in any medium, provided you give appropriate credit to the original author(s) and the source, provide a link to the Creative Commons license, and indicate if changes were made. 
In this paper, we will consider an anisotropic parabolic equation of the type

$$
u_{t}=\sum_{i=1}^{N}\left(a_{i}(x)\left|u_{x_{i}}\right|^{p_{i}(x)-2} u_{x_{i}}\right)_{x_{i}}, \quad(x, t) \in Q_{T} .
$$

We denote that

$$
p_{i}^{+}=\max _{x \in \bar{\Omega}} p_{i}(x), \quad p_{i}^{-}=\min _{x \in \bar{\Omega}} p_{i}(x)
$$

for any $i \in\{1,2, \ldots, N\}$ and denote that

$$
\begin{aligned}
& p_{0}=\min \left\{p_{1}^{-}, p_{2}^{-}, \ldots, p_{N-1}^{-}, p_{N}^{-}\right\}, \quad p_{0}>1, \\
& p^{0}=\max \left\{p_{1}^{+}, p_{2}^{+}, \ldots, p_{N-1}^{+}, p_{N}^{+}\right\} .
\end{aligned}
$$

If $a_{i}(x) \equiv 1$, the existence of a weak solution was proved in [9]. Also, one can refer to the excellent papers [10-12].

Let $I=\left\{i_{1}, i_{2}, \ldots, i_{k}\right\} \subset\{1,2, \ldots, N\}, J=\left\{j_{1}, j_{2}, \ldots, j_{l}\right\} \subset\{1,2, \ldots, N\}, k+l=N, I \cap J=\varnothing$. Not only we assume that $a_{i}(x) \in C(\bar{\Omega})$, and when $x \in \Omega, a_{i}(x)>0$, but we also assume that

$$
\begin{aligned}
& a_{i_{1}}(x) \geq c_{1}>0, \quad a_{i_{2}}(x) \geq c_{2}>0, \quad \ldots, \quad a_{i_{k}}(x) \geq c_{k}>0, \quad x \in \bar{\Omega}, \\
& a_{j_{1}}(x)=0, \quad a_{j_{2}}(x)=0, \quad \ldots, \quad a_{j_{l}}(x)=0, \quad x \in \partial \Omega .
\end{aligned}
$$

Besides the initial value condition (1.2), instead of the usual boundary value condition (1.3), by assumptions (1.5)-(1.6), only a partial boundary value condition

$$
u(x, t)=0, \quad(x, t) \in \Sigma_{1} \times(0, T)
$$

should be imposed. To see that, let us give a simple example to show what $\Sigma_{1}$ is. Let $N=2$, $p_{1}(x)=p_{2}(x) \equiv p(x)$, the domain $\Omega$ be a square,

$$
\Omega=\left\{\left(x_{1}, x_{2}\right): 0<x_{1}<1,0<x_{2}<1\right\} .
$$

Consider the equation

$$
u_{t}=\frac{\partial}{\partial x_{1}}\left(a_{1}(x)\left|u_{x_{1}}\right|^{p(x)-2} u_{x_{1}}\right)+\frac{\partial}{\partial x_{2}}\left(\left|u_{x_{1}}\right|^{p(x)-2} u_{x_{2}}\right) .
$$

Then we conjecture that

$$
\Sigma_{1}=\left\{\left(x_{1}, x_{2}\right): 0<x_{1}<1, x_{2}=0\right\} \cup\left\{\left(x_{1}, x_{2}\right): 0<x_{1}<1, x_{2}=1\right\} .
$$

This conjecture was proved in [13] recently.

However, in general, it is difficult to depict out the geometric character of $\Sigma_{1}$. We have tried to depict out $\Sigma_{1}$ by the Fichera function in [14], but it seems not so successful. In this short paper,

$$
\Sigma_{1}=\left\{x \in \partial \Omega: \frac{\left|\left(\prod_{j=1}^{l} a_{j}(x)\right)_{x_{i}}\right|^{p_{i_{r}}(x)}}{\left[\prod_{j=1}^{l} a_{j}(x)\right]^{p_{i_{r}(x)}-1}} \neq 0\right\},
$$


we will study the well-posedness of the equation basing on the partial boundary value condition (1.7). Also, we denote that

$$
\Sigma_{2}=\partial \Omega \backslash \Sigma_{1}
$$

Definition 1.1 A function $u(x, t)$ is said to be a weak solution of equation (1.4) with the initial value condition (1.2) if

$$
u \in L^{\infty}\left(Q_{T}\right), \quad \frac{\partial u}{\partial t} \in L^{2}\left(Q_{T}\right), \quad a_{i}(x)\left|u_{x_{i}}\right|^{p_{i}(x)} \in L^{2}\left(0, T ; L^{1}(\Omega)\right),
$$

and for any function $\varphi_{1} \in C_{0}^{1}\left(Q_{T}\right), \varphi_{2} \in L^{\infty}\left(Q_{T}\right)$ and $\varphi_{2 x_{i}} \in L^{2}\left(0, T ; W_{\text {loc }}^{1, p_{i}(x)}(\Omega)\right)$ such that

$$
\iint_{Q_{T}}\left[\frac{\partial u}{\partial t}\left(\varphi_{1} \varphi_{2}\right)+\sum_{i=1}^{N} a_{i}(x)\left|u_{x_{i}}\right|^{p_{i}(x)-2} u_{x_{i}}\left(\varphi_{1} \varphi_{2}\right)_{x_{i}}\right] d x d t=0 .
$$

The initial value condition (1.2) is satisfied in the sense of

$$
\lim _{t \rightarrow 0} \int_{\Omega}\left|u(x, t)-u_{0}(x)\right| d x=0 .
$$

Besides, if the partial boundary value condition (1.7) is satisfied in the sense of the trace, then we say that there is a weak solution of the initial-boundary value problem (1.4)-(1.2)(1.7).

In this paper, we first study the existence of the weak solution.

Theorem 1.2 If $p_{0}>1, a_{i}(x)$ satisfies conditions (1.5), (1.6),

$$
u_{0} \in L^{\infty}(\Omega), \quad u_{0 x_{i}} \in L^{p_{i}(x)}(\Omega),
$$

then there is a solution of equation (1.4) with the initial value (1.2). Moreover, if for every $1 \leq r \leq l, \int_{\Omega} a_{j_{r}}^{-1 /\left(p_{j_{r}}(x)-1\right)}(x) d x<\infty$, then the initial-boundary value problem (1.4)-(1.2)(1.7) has a solution.

Here, $L^{p_{i}(x)}(\Omega)$ is the variable exponent space, its definition is given in Section 2.

Secondly, we will study the stability of weak solutions to the initial-boundary value problem (1.4)-(1.2)-(1.7).

Theorem 1.3 If $u$ and $v$ are two solutions of equation (1.4) with the same partial boundary value condition (1.7) and with the initial values $u_{0}(x), v_{0}(x)$, respectively, if $l>1$ and for every $1 \leq r \leq l$,

$$
n\left(\int_{\Omega \backslash \Omega_{n}} a_{j_{r}}(x)\left|\left(\prod_{s=1}^{l} a_{j_{s}}(x)\right)_{x_{j r}}\right|^{p_{j r}(x)} d x\right)^{\frac{1}{p_{j r}^{+}}} \leq c,
$$

then

$$
\int_{\Omega}|u(x, t)-v(x, t)| d x \leq \int_{\Omega}\left|u_{0}(x)-v_{0}(x)\right| d x .
$$

Here, $\Omega_{n}=\left\{x \in \Omega: \prod_{s=1}^{l} a_{j_{s}}(x)>\frac{1}{n}\right\}$. 
If $l=1$ in Theorem 1.3, without loss of generality, we may assume that

$$
a_{1}(x)=0, \quad x \in \partial \Omega
$$

while $a_{i}(x)>0$ for $i>1$. Then we have the following.

Theorem 1.4 If (1.16) is true and $a_{i}(x)>0$ for $i>1, u$ and $v$ are two solutions of equation (1.4) with the same partial boundary value condition (1.7) and with the initial values $u_{0}(x)$, $v_{0}(x)$, respectively, iffor every $i \geq 2$

$$
n\left(\int_{\Omega_{n}}\left|a_{1 x_{i}}(x)\right|^{p_{i}(x)} d x\right)^{\frac{1}{p_{i}^{+}}} \leq c
$$

and

$$
n\left(\int_{\Omega \backslash \Omega_{n}} a_{1}(x)\left|a_{1 x_{1}}(x)\right|^{p_{1}(x)} d x\right)^{\frac{1}{p_{1}^{+}}} \leq c
$$

then the stability of weak solutions (1.15) is true. Here, $a_{1 x_{1}}(x)=\frac{\partial a_{1}(x)}{\partial x_{1}}, a_{1 x_{i}}(x)=\frac{\partial a_{1}(x)}{\partial x_{i}}$ as usual, $\Omega_{n}=\left\{x \in \Omega: a_{1}(x)>\frac{1}{n}\right\}$.

At the end of the introduction, we would like to suggest that there are many papers devoted to the anisotropic elliptic equations, for examples, one can see [15-25] and the references therein. For example, Fu and Shan studied the problem of removable isolated singularities for elliptic equations with variable exponents in [23]. They gave a sufficient condition for removability of the isolated singular point for the equations in $W^{1, p(x)}(\Omega)$. Cencelj and Repovš studied the perturbation by a critical term and a superlinear subcritical nonlinearity of a quasilinear elliptic equation containing a singular potential in [24]. By means of variational arguments and a version of the concentration-compactness principle in the singular case, they proved the existence of solutions for positive values of the parameter under the principal eigenvalue of the associated singular eigenvalue problem. Konaté and Ouaro studied nonlinear anisotropic problems with bounded Radon diffuse measure and variable exponent in [25]. They proved the existence and uniqueness of an entropy solution. By the way, the definition of weak solutions and the method used in [13] are different from the ones in this paper. Moreover, only the case when the domain is the $n$-dimensional unit cube is considered in [13], and the diffusion coefficient $a_{i}(x)=a_{i}\left(x_{i}\right)$ is restricted only dependent on the single variable $x_{i}$.

\section{The existence}

We firstly give some basic concepts about the exponent variable spaces.

1. $L^{p(x)}(\Omega)$ space.

$$
\begin{aligned}
L^{p(x)}(\Omega)= & \{u: u \text { is a measurable real-valued function, } \\
& \left.\int_{\Omega}|u(x)|^{p(x)} d x<\infty\right\} .
\end{aligned}
$$


The space $L^{p(x)}(\Omega)$ is equipped with the following Luxemburg norm:

$$
|u|_{L^{p(x)}}(\Omega)=\inf \left\{\lambda>0: \int_{\Omega}\left|\frac{u(x)}{\lambda}\right|^{p(x)} d x \leq 1\right\}
$$

The space $\left(L^{p(x)}(\Omega),|\cdot|_{L^{p(x)}(\Omega)}\right)$ is a separable, uniformly convex Banach space.

2. $W^{1, p(x)}(\Omega)$ space.

$$
W^{1, p(x)}(\Omega)=\left\{u \in L^{p(x)}(\Omega):|\nabla u| \in L^{p(x)}(\Omega)\right\}
$$

endowed with the following norm:

$$
|u|_{W^{1, p(x)}}=|u|_{L^{p(x)}(\Omega)}+|\nabla u|_{L^{p(x)}(\Omega)}, \quad \forall u \in W^{1, p(x)}(\Omega) .
$$

We use $W_{0}^{1, p(x)}(\Omega)$ to denote the closure of $C_{0}^{\infty}(\Omega)$ in $W^{1, p(x)}$.

Lemma $2.1([26-28])$ The spaces $\left(L^{p(x)}(\Omega),|\cdot|_{L^{p(x)}(\Omega)}\right),\left(W^{1, p(x)}(\Omega),|\cdot|_{W^{1, p(x)}(\Omega)}\right)$ and $W_{0}^{1, p(x)}(\Omega)$ are reflexive Banach spaces.

Lemma 2.2 ([13]) If, for any given $i \in\{1,2, \ldots, N\}, \int_{\Omega} a_{i}^{-\frac{1}{p_{i}(x)-1}}(x) d x<\infty$, then

$$
\int_{\Omega}\left|u_{x_{i}}\right| d x \leq c
$$

By this lemma, one can see that if $a_{i}(x)$ satisfies (1.5), (1.6) and if for every $1 \leq r \leq l$, $\int_{\Omega} a_{j_{r}}^{-1 /\left(p_{r}(x)^{-1)}\right.}(x) d x<\infty$, then (2.1) is satisfied. Thus, we can define the trace of $u$ on the boundary $\partial \Omega$.

Proof of Theorem 1.2 Consider the partially regularized equation

$$
u_{t}=\sum_{i=1}^{N}\left(a_{i}(x)\left|u_{x_{i}}\right|^{p_{i}(x)-2} u_{x_{i}}\right)_{x_{i}}+\varepsilon \Delta u, \quad(x, t) \in Q_{T}
$$

with the initial boundary conditions

$$
\begin{aligned}
& u(x, 0)=u_{0 \varepsilon}(x), \quad x \in \Omega, \\
& u(x, t)=0, \quad(x, t) \in \partial \Omega \times(0, T) .
\end{aligned}
$$

Here, we let $u_{0 \varepsilon}(x) \in C_{0}^{\infty}(\Omega)$ and strongly convergent to $u_{0}(x)$ in $W_{0}^{1, p^{0}}(\Omega)$.

Since $a_{i}(x)$ satisfies (1.5) and (1.6), similar to the proof of the usual evolutionary $p$ Laplacian equation, we can prove that there is a solution $u_{\varepsilon} \in L^{2}\left(0, T ; W_{0}^{1, p_{0}}(\Omega)\right)$ of the initial-boundary value problem (2.2)-(2.4), which satisfies

$$
\left\|u_{\varepsilon}\right\|_{L^{\infty}\left(Q_{T}\right)} \leq c, \quad\left\|u_{\varepsilon t}\right\|_{L^{2}\left(Q_{T}\right)} \leq c
$$


Multiplying (2.1) by $u_{\varepsilon}$ and integrating it over $Q_{T}$, we have

$$
\begin{aligned}
& \frac{1}{2} \int_{\Omega} u_{\varepsilon}^{2} d x+\sum_{i=1}^{n} \iint_{Q_{T}} a_{i}(x)\left|u_{\varepsilon x_{i}}\right|^{p_{i}(x)} d x d t+\varepsilon \iint_{Q_{T}}\left|\nabla u_{\varepsilon}\right|^{2} d x d t \\
& \quad=\frac{1}{2} \int_{\Omega} u_{0}^{2}(x) d x,
\end{aligned}
$$

then

$$
\varepsilon \iint_{Q_{T}}\left|\nabla u_{\varepsilon}\right|^{2} d x \leq c
$$

and

$$
\sum_{i=1}^{N} \iint_{Q_{T}} a_{i}(x)\left|u_{\varepsilon x_{i}}\right|^{p_{i}(x)} d x d t \leq c
$$

Hence, by (2.5), (2.7), (2.8), there exists a function $u$ and an $n$-dimensional vector $\vec{\zeta}=$ $\left(\zeta_{1}, \ldots, \zeta_{n}\right)$ satisfying that $\vec{\zeta}=\left(\zeta_{1}, \ldots, \zeta_{n}\right)$

$$
u \in L^{\infty}\left(Q_{T}\right), \quad \frac{\partial u}{\partial t} \in L^{2}\left(Q_{T}\right), \quad \zeta_{i} \in L^{1}\left(0, T ; L^{\frac{p_{i}(x)}{p_{i}(x)-1}}(\Omega)\right),
$$

and $u_{\varepsilon} \rightarrow u$ a.e. $\in Q_{T}$,

$$
\begin{aligned}
& u_{\varepsilon} \rightarrow u, \quad \text { weakly star in } L^{\infty}\left(Q_{T}\right), \\
& u_{\varepsilon} \rightarrow u, \quad \text { in } L^{2}\left(0, T ; L_{\mathrm{loc}}^{r}(\Omega)\right), \\
& \frac{\partial u_{\varepsilon}}{\partial t} \rightarrow \frac{\partial u}{\partial t} \quad \text { in } L^{2}\left(Q_{T}\right), \\
& \varepsilon \nabla u_{\varepsilon} \rightarrow 0, \quad \text { in } L^{2}\left(Q_{T}\right), \\
& a_{i}(x)\left|u_{\varepsilon x_{i}}\right|^{p_{i}(x)-2} u_{\varepsilon x_{i}} \rightarrow \zeta_{i} \quad \text { in } L^{1}\left(0, T ; L^{\frac{p_{i}(x)}{p_{i}(x)-1}}(\Omega)\right) .
\end{aligned}
$$

Here, $r<\frac{N p_{0}}{N-p^{0}}$.

Now, similar to the general evolutionary $p$-Laplacian equation, we are able to prove that (the details are omitted here)

$$
\lim _{t \rightarrow 0} \int_{\Omega}\left|u(x, t)-u_{0}(x)\right| d x=0
$$

and

$$
\sum_{i=1}^{N} \iint_{Q_{T}} a_{i}(x)\left|u_{x_{i}}\right|^{p_{i}(x)-2} u_{x_{i}} \varphi_{x_{i}} d x d t=\sum_{i=1}^{N} \iint_{Q_{T}} \zeta_{i}(x) \varphi_{x_{i}} d x d t
$$

for any function $\varphi \in C_{0}^{1}\left(Q_{T}\right)$. By a process of the limit [8], we can show that (2.9) is also true for any $\varphi=\varphi_{1} \varphi_{2}$, where $\varphi_{1} \in C_{0}^{1}\left(Q_{T}\right), \varphi_{2} \in L^{\infty}$ and $\varphi_{2 x_{i}} \in L^{2}\left(0, T ; W_{\text {loc }}^{1, p_{i}(x)}(\Omega)\right)$. Then 
$u$ satisfies equation (1.4) with the initial value (1.2) in the sense of Definition 1.1. At last, according to Lemma 2.2, the partial boundary value condition (1.7) is satisfied in the sense of trace. Theorem 1.2 is proved.

\section{The stability}

\section{Lemma 3.1 ([26-28])}

(i) $p(x)$-Hölder's inequality. Let $q_{1}(x)$ and $q_{2}(x)$ be real functions with $\frac{1}{q_{1}(x)}+\frac{1}{q_{2}(x)}=1$ and $q_{1}(x)>1$. Then the conjugate space of $L^{q_{1}(x)}(\Omega)$ is $L^{q_{2}(x)}(\Omega)$. And, for any $u \in L^{q_{1}(x)}(\Omega)$ and $v \in L^{q_{2}(x)}(\Omega)$, we have

$$
\left|\int_{\Omega} u v d x\right| \leq 2|u|_{L^{q_{1}(x)}(\Omega)}|v|_{L^{q_{2}(x)(\Omega)}}
$$

(ii)

$$
\begin{aligned}
& |u|_{L^{p(x)}(\Omega)}=1, \text { then } \int_{\Omega}|u|^{p(x)} d x=1, \\
& |u|_{L^{p(x)}}(\Omega)>1, \quad \text { then }|u|_{L^{p(x)}}^{p^{-}} \leq \int_{\Omega}|u|^{p^{(x)}} d x \leq|u|_{L^{p(x)}}^{p^{+}}, \\
& |u|_{L^{p(x)}}(\Omega)<1, \quad \text { then }|u|_{L^{p(x)}}^{p^{+}} \leq \int_{\Omega}|u|^{p^{(x)}} d x \leq|u|_{L^{p(x)}}^{p^{-}} .
\end{aligned}
$$

Now, we will prove Theorem 1.3. For any given positive integer $n$, let $g_{n}(s)$ be an odd function, and

$$
g_{n}(s)= \begin{cases}1, & s>\frac{1}{n}, \\ n^{2} s^{2} \mathrm{e}^{1-n^{2} s^{2}}, & 0 \leq s \leq \frac{1}{n} .\end{cases}
$$

Clearly,

$$
\lim _{n \rightarrow 0} g_{n}(s)=\operatorname{sign}(s), \quad s \in(-\infty,+\infty)
$$

Proof of Theorem 1.3 Let $u$ and $v$ be two weak solutions of equation (1.4) with the initial values $u(x, 0), v(x, 0)$, respectively.

Let $\Omega_{n}=\left\{x \in \Omega: \prod_{r=1}^{l} a_{j_{r}}(x)>\frac{1}{n}\right\}$, and

$$
\phi_{n}(x)= \begin{cases}1, & \text { if } x \in \Omega_{n}, \\ n \prod_{r=1}^{l} a_{j_{r}}(x), & \text { if } x \in \Omega \backslash \Omega_{n} .\end{cases}
$$

Obviously, $\phi_{n x_{i}}=n\left(\prod_{r=1}^{l} a_{j_{r}}(x)\right)_{x_{i}}$ when $x \in \Omega \backslash \Omega_{n}$, in other places, it is identical to zero. We can choose $\varphi_{1}=\chi_{[\tau, s]} \phi_{n}, \varphi_{2}=g_{n}(u-v), \varphi=\chi_{[\tau, s]} \phi_{n} g_{n}(u-v)$ as the test function, then

$$
\begin{aligned}
& \int_{\tau}^{s} \int_{\Omega} \phi_{n} g_{n}(u-v) \frac{\partial(u-v)}{\partial t} d x d t \\
& +\sum_{i=1}^{N} \int_{\tau}^{s} \int_{\Omega} a_{i}(x)\left(\left|u_{x_{i}}\right|^{p_{i}(x)-2} u_{x_{i}}-\left|v_{x_{i}}\right|^{p_{i}(x)-2} v_{x_{i}}\right)\left(u_{x_{i}}-v_{x_{i}}\right) g_{n}^{\prime}(u-v) \phi_{n}(x) d x d t \\
& +\sum_{r=1}^{k} \int_{\tau}^{s} \int_{\Omega} a_{i_{r}}(x)\left(\left|u_{x_{i_{r}}}\right|^{p_{i r}(x)-2} u_{x_{i_{r}}}-\left|v_{x_{i_{r}}}\right|^{p_{i_{r}}(x)-2} v_{x_{i_{r}}}\right) g_{n}(u-v) \phi_{n x_{i_{r}}} d x d t
\end{aligned}
$$




$$
+\sum_{r=l}^{l} \int_{\tau}^{s} \int_{\Omega} a_{j_{r}}(x)\left(\left|u_{x_{j r}}\right|^{p_{j_{r}}(x)-2} u_{x_{j_{r}}}-\left|v_{x_{j r}}\right|^{p_{j_{r}}(x)-2} v_{x_{j_{r}}}\right) g_{n}(u-v) \phi_{n x_{j r}} d x d t
$$

$=0$.

In the first place, as usual, we have

$$
\int_{\Omega} a_{i}(x)\left(\left|u_{x_{i}}\right|^{p_{i}(x)-2} u_{x_{i}}-\left|v_{x_{i}}\right|^{p_{i}(x)-2} v_{x_{i}}\right)\left(u_{x_{i}}-v_{x_{i}}\right) g_{n}^{\prime}(u-v) \phi_{n}(x) d x \geq 0,
$$

and since $u_{t} \in L^{2}\left(Q_{T}\right)$, using the Lebesgue dominated theorem, we have

$$
\begin{aligned}
& \lim _{\eta \rightarrow 0} \int_{\tau}^{s} \int_{\Omega} \phi_{n}(x) g_{n}(u-v) \frac{\partial(u-v)}{\partial t} d x d t \\
& \quad=\int_{\Omega}|u-v|(x, s) d x-\int_{\Omega}|u-v|(x, \tau) d x
\end{aligned}
$$

In the second place, we deal with the third term on the left-hand side of (3.3). For simplicity, in what follows, we denote $\prod_{r=1}^{l} a_{j_{r}}(x)$ as $\prod_{j=1}^{l} a_{j}(x)$,

$$
\begin{aligned}
& \left|\int_{\Omega} a_{i_{r}}(x)\left(\left|u_{x_{i_{r}}}\right|^{p_{i_{r}}(x)-2} u_{x_{i_{r}}}-\left|v_{x_{i_{r}}}\right|^{p_{i_{r}}(x)-2} v_{x_{i_{r}}}\right) g_{n}(u-v) \phi_{n x_{i_{r}}} d x\right| \\
& =\left|\int_{\Omega \backslash \Omega_{n}} a_{i_{r}}(x)\left(\left|u_{x_{i_{r}}}\right|^{p_{i_{r}}(x)-2} u_{x_{i_{r}}}-\left|v_{x_{i_{r}}}\right|^{p_{i_{r}}(x)-2} v_{x_{i_{r}}}\right) g_{n}(u-v) \phi_{n x_{i_{r}}} d x\right| \\
& \leq \int_{\Omega \backslash \Omega_{n}} a_{i_{r}}(x)\left(\left|u_{x_{i_{r}}}\right|^{p_{i_{r}}(x)-1}+\left|v_{x_{i_{r}}}\right|^{p_{i_{r}}(x)-1}\right)\left|g_{n}(u-v) \phi_{n x_{i_{r}}}\right| d x \\
& \leq \int_{\Omega \backslash \Omega_{n}}\left[n a_{i_{r}}(x) \prod_{j=1}^{l} a_{j}(x)\right]^{\frac{p_{i_{r}(x)}-1}{p_{i_{r}(x)}}}\left(\left|u_{x_{i_{r}}}\right|^{p_{i_{r}}(x)-1}+\left|v_{x_{i_{r}}}\right|^{p_{i_{r}}(x)-1}\right) \\
& \cdot\left(a_{i_{r}}(x)\right)^{\frac{1}{p_{i_{r}}(x)}}\left|g_{n}(u-v) \frac{n^{\frac{1}{p_{i_{r}}(x)}}\left[\prod_{j=1}^{l} a_{j}(x)\right]_{x_{i_{r}}}}{\left[\prod_{j=1}^{l} a_{j}(x)\right]^{\frac{p_{i_{r}(x)^{-1}}}{p_{i_{r}(x)}}}}\right| d x \\
& \leq\left(\int_{\Omega \backslash \Omega_{n}} n \prod_{j=1}^{l} a_{j}(x) a_{i_{r}}(x)\left(\left|u_{x_{i_{r}}}\right|^{p_{i_{r}}(x)}+\left|v_{x_{i_{r}}}\right|^{p_{i_{r}}(x)}\right) d x\right)^{\frac{1}{q_{i_{r}}^{1}}} \\
& \cdot\left(\int_{\Omega \backslash \Omega_{n}} n a_{i_{r}}(x)\left|g_{n}(u-v)\right|^{p_{i_{r}}(x)} \frac{\left|\left(\prod_{j=1}^{l} a_{j}(x)\right)_{x_{i_{r}}}\right|^{p_{i_{r}}(x)}}{\left[\prod_{j=1}^{l} a_{j}(x)\right]^{p_{i_{r}(x)}-1}} d x\right)^{\frac{1}{p_{i_{r}}^{1}}} \\
& \leq c\left(\int_{\Omega \backslash \Omega_{n}} a_{i_{r}}(x)\left(\left|u_{x_{i_{r}}}\right|^{p_{i_{r}}(x)}+\left|v_{x_{i_{r}}}\right|^{p_{i_{r}}(x)}\right) d x\right)^{\frac{1}{q_{i_{r}}^{1}}} \\
& \cdot\left(n \int_{\Omega \backslash \Omega_{n}}\left|g_{n}(u-v)\right|^{p_{i_{r}}(x)} \frac{\left|\left(\prod_{j=1}^{l} a_{j}(x)\right)_{x_{i_{r}}}\right|^{p_{i_{r}}(x)}}{\left[\prod_{j=1}^{l} a_{j}(x)\right]^{p_{i_{r}(x)}-1}} d x\right)^{\frac{1}{p_{i_{r}}^{1}}} .
\end{aligned}
$$

Here, $p_{i_{r}}^{1}=p_{i_{r}}^{+}$or $p_{i_{r}}^{-}$according to (ii) of Lemma 3.1. $q_{j_{r}}(x)=\frac{p_{j_{r}}(x)}{p_{j_{r}}(x)-1}, q_{i_{r}}^{1}$ has a similar sense. If we denote that

$$
\Omega_{n 1}=\left\{x \in \Omega \backslash \Omega_{n}: \operatorname{dist}\left(x, \Sigma_{2}\right)>\operatorname{dist}\left(x, \Sigma_{1}\right)\right\},
$$


Chan Boundary Value Problems ( 2017) 2017:166

Page 9 of 14

$$
\Omega_{n 2}=\left\{x \in \Omega \backslash \Omega_{n}: \operatorname{dist}\left(x, \Sigma_{2}\right) \leq \operatorname{dist}\left(x, \Sigma_{1}\right)\right\},
$$

then

$$
\begin{aligned}
& n \int_{\Omega \backslash \Omega_{n}}\left|g_{n}(u-v)\right|^{p_{i_{r}}(x)} \frac{\mid\left(\prod_{j=1}^{l} a_{j}(x)\right)_{x_{i_{r}}}{ }^{p_{i_{r}}(x)}}{\left[\prod_{j=1}^{l} a_{j}(x)\right]^{p_{i_{r}(x)}-1}} d x \\
& \leq n \int_{\Omega_{n 1}}\left|g_{n}(u-v)\right|^{p_{i_{r}}(x)} \frac{\left|\left(\prod_{j=1}^{l} a_{j}(x)\right)_{x_{i_{r}}}\right|^{p_{i_{r}}(x)}}{\left[\prod_{j=1}^{l} a_{j}(x)\right]^{p_{i_{r}(x)}-1}} d x \\
& \quad+n \int_{\Omega_{n 2}}\left|g_{n}(u-v)\right|^{p_{i_{r}}(x)} \frac{\left|\left(\prod_{j=1}^{l} a_{j}(x)\right)_{x_{i_{r}}}\right|^{p_{i_{r}}(x)}}{\left[\prod_{j=1}^{l} a_{j}(x)\right]^{p_{i_{r}(x)}-1}} d x .
\end{aligned}
$$

Since

$$
u=v=0, \quad x \in \Sigma_{1},
$$

by the definition of the trace, we have

$$
\begin{gathered}
\lim _{n \rightarrow \infty} n \int_{\Omega_{n 1}}\left|g_{n}(u-v)\right|^{p_{i_{r}}(x)} \frac{\left|\left(\prod_{j=1}^{l} a_{j}(x)\right)_{x_{i_{r}}}\right|^{p_{i_{r}}(x)}}{\left[\prod_{j=1}^{l} a_{j}(x)\right]^{p_{i_{r}(x)}-1}} d x \\
=\int_{\Sigma_{1}} \operatorname{sign}(u-v) \frac{\left|\left(\prod_{j=1}^{l} a_{j}(x)\right)_{x_{i_{r}}}\right|^{p_{i_{r}}(x)}}{\left[\prod_{j=1}^{l} a_{j}(x)\right]^{p_{i_{r}(x)}}-1} d \Sigma=0 .
\end{gathered}
$$

Moreover, since

$$
\begin{aligned}
& \frac{\left|\left(\prod_{j=1}^{l} a_{j}(x)\right)_{x_{i_{r}}}\right|^{p_{i_{r}}(x)}}{\left[\prod_{j=1}^{l} a_{j}(x)\right]^{p_{i_{r}(x)}-1}}=0, \quad x \in \Sigma_{2}, \\
& \lim _{n \rightarrow \infty} n \int_{\Omega_{n 2}}\left|g_{n}(u-v)\right|^{p_{i_{r}}(x)} \frac{\left|\left(\prod_{j=1}^{l} a_{j}(x)\right)_{x_{i_{r}}}\right|^{p_{i_{r}}(x)}}{\left[\prod_{j=1}^{l} a_{j}(x)\right]^{p_{i_{r}(x)}-1}} d x \\
& \quad \leq \lim _{n \rightarrow \infty} n \int_{\Omega_{n 2}} \frac{\left|\left(\prod_{j=1}^{l} a_{j}(x)\right)_{x_{i_{r}}}\right|^{p_{i_{r}}(x)}}{\left[\prod_{j=1}^{l} a_{j}(x)\right]^{p_{i_{r}(x)}-1}} d x \\
& \quad=\int_{\Sigma_{2}} \frac{\left|\left(\prod_{j=1}^{l} a_{j}(x)\right)_{x_{i_{r}}}\right|^{p_{i_{r}}(x)}}{\left[\prod_{j=1}^{l} a_{j}(x)\right]^{p_{i_{r}(x)}-1}} d \Sigma=0 .
\end{aligned}
$$

By (3.6)-(3.9), we conclude that

$$
\lim _{n \rightarrow 0}\left|\int_{\Omega} a_{i_{r}}(x)\left(\left|u_{x_{i_{r}}}\right|^{p_{i_{r}}(x)-2} u_{x_{i_{r}}}-\left|v_{x_{i_{r}}}\right|^{p_{i_{r}}(x)-2} v_{x_{i_{r}}}\right) g_{n}(u-v) \phi_{n x_{i_{r}}} d x\right|=0
$$

In the third place, we deal with the last term on the left-hand side of (3.3)

$$
\begin{aligned}
& \left|\int_{\Omega} a_{j_{r}}(x)\left(\left|u_{x_{j r}}\right|^{p_{j_{r}}(x)-2} u_{x_{j_{r}}}-\left|v_{x_{j r}}\right|_{p_{j_{r}}(x)-2} v_{x_{j_{r}}}\right) g_{n}(u-v) \phi_{n x_{j_{r}}} d x\right| \\
& \quad=\left|\int_{\Omega \backslash \Omega_{n}} a_{j_{r}}(x)\left(\left|u_{x_{j_{r}}}\right|^{p_{j_{r}}(x)-2} u_{x_{j_{r}}}-\left|v_{x_{j r}}\right| p_{j_{r}}(x)-2 v_{x_{j_{r}}}\right) g_{n}(u-v) \phi_{n x_{j r}} d x\right|
\end{aligned}
$$




$$
\begin{aligned}
& \leq \int_{\Omega \backslash \Omega_{n}} a_{j_{r}}(x)\left(\left|u_{x_{j r}}\right|_{p_{j r}(x)-1}+\mid v_{x_{j r}} p^{p_{j_{r}}(x)-1}\right)\left(\prod_{j=1}^{l} a_{j}(x)\right)_{x_{j r}} g_{n}(u-v) \mid d x \\
& \leq c\left(\int_{\Omega \backslash \Omega_{n}} a_{j_{r}}(x)\left|u_{x_{j r}}\right|^{p_{j r}(x)}+\left|v_{x_{j r}}\right|^{p_{j_{r}}(x)} d x\right)^{\frac{1}{q_{j r}^{+}}} \\
& \times n\left(\int_{\Omega \backslash \Omega_{n}} a_{j_{r}}(x)\left|\left(\prod_{j=1}^{l} a_{j}(x)\right)_{x_{i}}\right|^{p_{j r}(x)} d x\right)^{\frac{1}{p_{j_{r}}^{+}}} .
\end{aligned}
$$

Here, $q_{j_{r}}(x)=\frac{p_{j_{r}}(x)}{p_{j_{r}}(x)-1}, q_{j_{r}}^{+}=\max _{x \in \bar{\Omega}} q_{j_{r}}(x)$.

By assumption (1.14)

$$
n\left(\int_{\Omega \backslash \Omega_{n}} a_{j r}(x)\left|\left(\prod_{j=1}^{l} a_{j}(x)\right)_{x_{j r}}\right|^{p_{j r}(x)} d x\right)^{\frac{1}{p_{j r}^{+}}} \leq c .
$$

Then

$$
\lim _{n \rightarrow \infty}\left|\int_{\Omega} a_{j_{r}}(x)\left(\left|u_{x_{j r}}\right|^{p_{j_{r}}(x)-2} u_{x_{j_{r}}}-\left|v_{x_{j_{r}}}\right|^{p_{j_{r}}(x)-2} v_{x_{j r}}\right) g_{n}(u-v) \phi_{n x_{j r}} d x\right|=0 .
$$

Now, let $n \rightarrow \infty$ in (3.3). Then

$$
\int_{\Omega}|u(x, s)-v(x, s)| d x \leq \int_{\Omega}|u(x, \tau)-v(x, \tau)| d x
$$

by the arbitrariness of $\tau$, we have

$$
\int_{\Omega}|u(x, s)-v(x, s)| d x \leq \int_{\Omega}\left|u_{0}(x)-v_{0}(x)\right| d x .
$$

\section{The case of $I=1$}

Proof of Theorem 1.3 Let $u$ and $v$ be two weak solutions of equation (1.4) with the initial values $u(x, 0), v(x, 0)$, respectively. Since $a_{1}(x)$ satisfies (1.16) and for $i \geq 2, a_{i}(x)>0, x \in \bar{\Omega}$, we can let $\Omega_{n}=\left\{x \in \Omega: a_{1}(x)>\frac{1}{n}\right\}$ and

$$
\phi_{n}(x)= \begin{cases}1, & \text { if } x \in \Omega_{n}, \\ n a_{1}(x), & \text { if } x \in \Omega \backslash \Omega_{n} .\end{cases}
$$

Obviously, $\phi_{n x_{i}}=n a_{1 x_{i}}$ when $x \in \Omega \backslash \Omega_{n}$, in other places, it is identical to zero.

We can choose $\chi_{[\tau, s]} \phi_{n} g_{n}(u-v)$ as the test function, then

$$
\begin{aligned}
& \int_{\tau}^{s} \int_{\Omega} \phi_{n} g_{n}(u-v) \frac{\partial(u-v)}{\partial t} d x d t \\
& \quad+\sum_{i=1}^{N} \int_{\tau}^{s} \int_{\Omega} a_{i}(x)\left(\left|u_{x_{i}}\right|^{p_{i}(x)-2} u_{x_{i}}-\left|v_{x_{i}}\right|^{p_{i}(x)-2} v_{x_{i}}\right)\left(u_{x_{i}}-v_{x_{i}}\right) g_{n}^{\prime}(u-v) \phi_{n}(x) d x d t \\
& \quad+\int_{\tau}^{s} \int_{\Omega} a_{1}(x)\left(\left|u_{x_{1}}\right|^{p_{1}(x)-2} u_{x_{1}}-\left|v_{x_{1}}\right|^{p_{1}(x)-2} v_{x_{1}}\right) g_{n}(u-v) \phi_{n x_{1}} d x d t
\end{aligned}
$$




$$
+\sum_{i=2}^{N} \int_{\tau}^{s} \int_{\Omega} a_{i}(x)\left(\left|u_{x_{i}}\right|^{p_{i}(x)-2} u_{x_{i}}-\left|v_{x_{i}}\right|^{p_{i}(x)-2} v_{x_{i}}\right) g_{n}(u-v) \phi_{n x_{i}} d x d t
$$

$$
=0 \text {. }
$$

Certainly, we have

$$
\int_{\Omega} a_{i}(x)\left(\left|u_{x_{i}}\right|^{p_{i}(x)-2} u_{x_{i}}-\left|v_{x_{i}}\right|^{p_{i}(x)-2} v_{x_{i}}\right)\left(u_{x_{i}}-v_{x_{i}}\right) g_{n}^{\prime}(u-v) \phi_{n}(x) d x \geq 0
$$

and

$$
\begin{gathered}
\lim _{\eta \rightarrow 0} \int_{\tau}^{s} \int_{\Omega} \phi_{n}(x) \phi_{n} g_{n}(u-v) \frac{\partial(u-v)}{\partial t} d x d t \\
=\int_{\Omega}|u-v|(x, s) d x-\int_{\Omega}|u-v|(x, \tau) d x
\end{gathered}
$$

Now, we deal with the third term on the left-hand side of (4.2).

$$
\begin{aligned}
& \left|\int_{\Omega} a_{1}(x)\left(\left|u_{x_{1}}\right|^{p_{1}(x)-2} u_{x_{1}}-\left|v_{x_{1}}\right|^{p_{1}(x)-2} v_{x_{1}}\right) g_{n}(u-v) \phi_{n x_{1}} d x\right| \\
& =\left|\int_{\Omega \backslash \Omega_{n}} a_{1}(x)\left(\left|u_{x_{1}}\right|^{p_{1}(x)-2} u_{x_{1}}-\left|v_{x_{1}}\right|^{p_{1}(x)-2} v_{x_{1}}\right) g_{n}(u-v) \phi_{n x_{1}} d x\right| \\
& \leq \int_{\Omega \backslash \Omega_{n}} a_{1}(x)\left(\left|u_{x_{1}}\right|^{p_{1}(x)-1}+\left|v_{x_{1}}\right|^{p_{1}(x)-1}\right)\left|g_{n}(u-v) \phi_{n x_{1}}\right| d x \\
& \leq n \int_{\Omega \backslash \Omega_{n}} a_{1}(x)\left(\left|u_{x_{1}}\right|^{p_{1}(x)-1}+\left|v_{x_{1}}\right|^{p_{1}(x)-1}\right) \mid g_{n}(u-v)\left(a_{x_{1}}(x) \mid d x\right. \\
& \leq c n\left(\int_{\Omega \backslash \Omega_{n}} a_{1}(x)\left(\left|u_{x_{1}}\right|^{p_{1}(x)}+\left|v_{x_{1}}\right|^{p_{1}(x)}\right) d x\right)^{\frac{1}{q_{1}^{+}}} \\
& \times\left(\int_{\Omega \backslash \Omega_{n}} a_{1}(x)\left|g_{n}(u-v)\right|^{p_{1}(x)}\left|a_{x_{1}}(x)\right|^{p_{1}(x)} d x\right)^{\frac{1}{p_{1}^{+}}} \\
& \leq c n^{1-\frac{1}{p_{1}^{+}}-\frac{1}{q_{1}^{+}}}\left(n \int_{\Omega \backslash \Omega_{n}} a_{1}(x)\left(\left|u_{x_{1}}\right|^{p_{1}(x)}+\left|v_{x_{1}}\right|^{p_{1}(x)}\right) d x\right)^{\frac{1}{q_{1}^{+}}} \\
& \times\left(\int_{\Omega \backslash \Omega_{n}}\left|g_{n}(u-v)\right|^{p_{1}(x)}\left|a_{x_{1}}(x)\right|^{p_{1}(x)} d x\right)^{\frac{1}{p_{1}^{+}}} .
\end{aligned}
$$

If we denote that

$$
d_{n}=\sup _{x \in \Omega \backslash \Omega_{n}} \operatorname{dist}(x, \partial \Omega)
$$

and

$$
\Omega_{n 1}=\left\{x \in \Omega \backslash \Omega_{n}: \operatorname{dist}\left(x, \Sigma_{2}\right)>d_{n}\right\}, \quad \Omega_{n 2}=\left(\Omega \backslash \Omega_{n}\right) \backslash \overline{\Omega_{n 1}},
$$


then

$$
\begin{aligned}
& n\left(\int_{\Omega \backslash \Omega_{n}} a_{i_{r}}(x)\left|g_{n}(u-v)\right|^{p_{i_{r}}(x)}\left|\left(\prod_{j=1}^{l} a_{j}(x)\right)_{x_{i_{r}}}\right|^{p_{i_{r}}(x)} d x\right)^{\frac{1}{p_{i r}^{+}}} \\
& \quad \leq n\left(\int_{\Omega_{n 1}} a_{i_{r}}(x)\left|g_{n}(u-v)\right|^{p_{i_{r}}(x)}\left|\left(\prod_{j=1}^{l} a_{j}(x)\right)_{x_{i_{r}}}\right|^{p_{i_{r}(x)}} d x\right)^{\frac{1}{p_{i_{r}}^{+}}} \\
& \quad+n\left(\int_{\Omega_{n 2}} a_{i_{r}}(x)\left|g_{n}(u-v)\right|^{p_{i_{r}}(x)}\left|\left(\prod_{j=1}^{l} a_{j}(x)\right)_{x_{i_{r}}}\right|^{p_{i_{r}}(x)} d x\right)^{\frac{1}{p_{i_{r}}^{+}}} \\
& \quad \leq n\left(\int_{\Omega_{n 1}}\left|g_{n}(u-v)\right|^{p_{i_{r}}(x)} d x\right)^{\frac{1}{p_{i r}^{+}}} \\
& \quad+n\left(\int_{\Omega_{n 2}} a_{i_{r}}(x)\left|\left(\prod_{j=1}^{l} a_{j}(x)\right)_{x_{i_{r}}}\right|^{p_{i_{r}}(x)} d x\right)^{\frac{1}{p_{i_{r}}^{+}}} .
\end{aligned}
$$

By the definition of the trace, we have

$$
\lim _{n \rightarrow \infty} n \int_{\Omega_{n 1}}\left|g_{n}(u-v)\right|^{p_{i r}} d x=0
$$

Moreover, since $a_{i}(x) \in C^{1}(\bar{\Omega})$ and $l>1$, by (1.6), we always have

$$
\left.\left(\prod_{j=1}^{l} a_{j}(x)\right)_{x_{i_{r}}}\right|_{\partial \Omega}=0 .
$$

Thus, by the fact that $a_{i}(x) \in C^{1}(\bar{\Omega})$, we get

$$
\begin{aligned}
& \lim _{n \rightarrow \infty} n\left(\int_{\Omega_{n 2}} a_{i_{r}}(x)\left|\left(\prod_{j=1}^{l} a_{j}(x)\right)_{x_{i_{r}}}\right|^{p_{i_{r}}} d x\right)^{\frac{1}{p_{i_{r}}^{+}}} \\
& \leq c \max _{x \in \Sigma_{2}}\left|\left(\prod_{j=1}^{l} a_{j}(x)\right)_{x_{i_{r}}}\right|=0 .
\end{aligned}
$$

By (4.6)-(4.10), we conclude that

$$
\lim _{n \rightarrow 0}\left|\int_{\Omega} a_{i_{r}}(x)\left(\left|u_{x_{i_{r}}}\right|^{p_{i_{r}}(x)-2} u_{x_{i_{r}}}-\left|v_{x_{i_{r}}}\right|_{i_{r}(x)-2} v_{x_{i_{r}}}\right) g_{n}(u-v) \phi_{n x_{i_{r}}} d x\right|=0 .
$$

In the third place, we deal with the last term on the left-hand side of (4.2)

$$
\begin{aligned}
& \left|\int_{\Omega} a_{j_{r}}(x)\left(\left|u_{x_{j r}}\right|^{p_{j_{r}}(x)-2} u_{x_{j r}}-\left|v_{x_{j r}}\right| p_{j_{r}}(x)-2 v_{x_{j_{r}}}\right) g_{n}(u-v) \phi_{n x_{j r}} d x\right| \\
& \quad=\left|\int_{\Omega \backslash \Omega_{n}} a_{j_{r}}(x)\left(\left|u_{x_{j r}}\right|^{p_{j_{r}}(x)-2} u_{x_{j_{r}}}-\left|v_{x_{j_{r}}}\right| p_{j_{r}}(x)-2 v_{x_{j r}}\right) g_{n}(u-v) \phi_{n x_{j r}} d x\right|
\end{aligned}
$$




$$
\begin{aligned}
& \leq \int_{\Omega \backslash \Omega_{n}} a_{j_{r}}(x)\left(\left|u_{x_{j r}}\right|_{p_{j r}(x)-1}+\mid v_{x_{j r}} p^{p_{j_{r}}(x)-1}\right)\left(\prod_{j=1}^{l} a_{j}(x)\right)_{x_{j r}} g_{n}(u-v) \mid d x \\
& \leq c\left(\int_{\Omega \backslash \Omega_{n}} a_{j_{r}}(x)\left(\left|u_{x_{j r}}\right|_{j_{r}(x)}+\left|v_{x_{j r}}\right|_{j_{r}}(x)\right) d x\right)^{\frac{1}{q_{j r}^{+}}} \\
& \times n\left(\int_{\Omega \backslash \Omega_{n}} a_{j_{r}}(x)\left|\left(\prod_{j=1}^{l} a_{j}(x)\right)_{x_{i}}\right|^{p_{j_{r}}(x)} d x\right)^{\frac{1}{p_{j r}^{+}}} .
\end{aligned}
$$

Here, $q_{j_{r}}(x)=\frac{p_{j_{r}}(x)}{p_{j_{r}}(x)-1}, q_{j_{r}}^{+}=\max _{x \in \bar{\Omega}} q_{j_{r}}(x)$.

By assumption (1.14),

$$
n\left(\int_{\Omega \backslash \Omega_{n}} a_{j_{r}}(x)\left|\left(\prod_{j=1}^{l} a_{j}(x)\right)_{x_{j_{r}}}\right|^{p_{j_{r}}(x)} d x\right)^{\frac{1}{p_{j_{r}}^{+}}} \leq c .
$$

Then

$$
\lim _{n \rightarrow \infty}\left|\int_{\Omega} a_{j_{r}}(x)\left(\left|u_{x_{j_{r}}}\right|^{p_{j_{r}}(x)-2} u_{x_{j_{r}}}-\left|v_{x_{j_{r}}}\right|^{p_{j_{r}}(x)-2} v_{x_{j_{r}}}\right) g_{n}(u-v) \phi_{n x_{j_{r}}} d x\right|=0 .
$$

Now, let $n \rightarrow \infty$ in (4.2). Then

$$
\int_{\Omega}|u(x, s)-v(x, s)| d x \leq \int_{\Omega}|u(x, \tau)-v(x, \tau)| d x,
$$

by the arbitrariness of $\tau$, we have

$$
\int_{\Omega}|u(x, s)-v(x, s)| d x \leq \int_{\Omega}\left|u_{0}(x)-v_{0}(x)\right| d x .
$$

\section{Conclusion}

The anisotropic parabolic equation is considered in this paper. If in some directions the diffusion coefficients are degenerate on the boundary, while in other directions they are not degenerate, how to give a suitable partial boundary value condition to match the equation was studied by the author in [13]. If a partial boundary value condition is imposed, only when the domain is an $\mathrm{N}$-dimensional cube, the stability of weak solutions is proved [13]. This short paper solves the problem when the domain is a usual bounded domain, gives a complete supplement of the paper [13].

\section{Acknowledgements}

The paper is supported by NSF of Fujian Province (Grant No. 2015J1092), supported by SF of Xiamen University of Technology (Grant No. XYK201448), China.

\section{Competing interests}

The author declares that they have no competing interests.

\section{Authors' contributions}

All authors read and approved the final manuscript.

\section{Publisher's Note}

Springer Nature remains neutral with regard to jurisdictional claims in published maps and institutional affiliations. 


\section{References}

1. Ruzicka, M: Electrorheological Fluids: Modeling and Mathematical Theory. Lecture Notes in Math., vol. 1748. Springer Berlin (2000)

2. Acerbi, E, Mingione, G: Regularity results for stationary electrorheological fluids. Arch. Ration. Mech. Anal. 164 213-259 (2002)

3. Antontsev, S, Shmarev, S: Anisotropic parabolic equations with variable nonlinearity. Publ. Mat. 53, 355-399 (2009)

4. Antontsev, S, Shmarev, S: Parabolic equations with double variable nonlinearities. Math. Comput. Simul. 81, 2018-2032 (2011)

5. Lian, S, Gao, W, Yuan, H, Cao, C: Existence of solutions to an initial Dirichlet problem of evolutional $p(x)$-Laplace equations. Ann. Inst. Henri Poincaré, Anal. Non Linéaire 29, 377-399 (2012)

6. Zhan, $\mathrm{H}$, Wen, J: Evolutionary $p(x)$-Laplacian equation free from the limitation of the boundary value. Electron. J. Differ. Equ. 2016, 143 (2016)

7. Zhan, H: The stability of evolutionary $p(x)$-Laplacian equation. Bound. Value Probl. 2017, 13 (2017). doi:10.1186/s13661-016-0742-0

8. Zhan, H, Xu, B: A new kind of weak solution of non-Newtonian fluid equation. J. Funct. Spaces 2017, 7916730 (2017). doi:10.1155/2017/7916730

9. Antontsev, S, Shmarev, S: Existence and uniqueness for doubly nonlinear parabolic equations with nonstandard growth conditions. Differ. Equ. Appl. 4(1), 67-94 (2012)

10. Tersenov Alkis, S: The one dimensional parabolic $p(x)$-Laplace equation. Nonlinear Differ. Equ. Appl. 23, 27 (2016). doi:10.1007/s00030-016-0377-y

11. Tersenov Alkis, S, Tersenov Aris, S: Existence of Lipschitz continuous solutions to the Cauchy-Dirichlet problem for anisotropic parabolic equations. J. Funct. Anal. 272, 3965-3986 (2017)

12. Aramaki, J: Hölder continuity with exponent $(1+\alpha) / 2$ in the time variable for solutions of parabolic equations. Electron. J. Differ. Equ 2015, 96 (2015)

13. Zhan, $\mathrm{H}$ : The stability of the anisotropic parabolic equation with the variable exponent. Bound. Value Probl. 2017, 134 (2017) doi:10.1186/s13661-017-0868-8

14. Zhan, $\mathrm{H}$ : The boundary value condition of an evolutionary $p(x)$-Laplacian equation. Bound. Value Probl. 2015, 112 (2015). doi:10.1186/s13661-015-0377-6

15. Di Nardo, R, Feo, F: Existence and uniqueness for nonlinear anisotropic elliptic equations. Arch. Math. 102, 141-153 (2014)

16. Mihailescu, M, Pucci, P, Radulescu, V: Eigenvalue problems for anisotropic quasilinear elliptic equations with variable exponent. J. Math. Anal. Appl. 340, 687-698 (2008)

17. Radulescu, V: Nonlinear elliptic equations with variable exponent: old and new. Nonlinear Anal., Theory Methods Appl. 121, 336-369 (2015)

18. Di Castro, A: Anisotropic elliptic problems with natural growth terms. Manuscr. Math. 135, 521-543 (2011)

19. El Hamidi, A, Vétois, J: Sharp Sobolev asymptotics for critical anisotropic equations. Arch. Ration. Mech. Anal. 192, $1-26$ (2009)

20. Vétois, J: The blow-up of critical anisotropic equations with critical directions. Nonlinear Differ. Equ. Appl. 18, 173-197 (2011)

21. Fragalà, I, Gazzola, F, Kawohl, B: Existence and nonexistence results for anisotropic quasilinear equations. Ann. Inst. Henri Poincaré, Anal. Non Linéaire 21, 715-734 (2004)

22. Radulescu, $V$, Repovs, D: Combined effects in nonlinear problems arising in the study of anisotropic continuous media. Nonlinear Anal. 75, 1524-1530 (2012)

23. Fu, F, Shan, Y: On the removability of isolated singular points for elliptic equations involving variable exponent. Adv. Nonlinear Anal. 5, 121-132 (2015)

24. Cencelj, M, Repovš, D, Virk, Ž: Multiple perturbations of a singular eigenvalue problem. Nonlinear Anal. 119, 37-45 (2015)

25. Konaté, I, Ouaro, S: Good Radon measure for anisotropic problems with variable exponent. Electron. J. Differ. Equ. 2016, 221 (2016)

26. Zhikov, W: On the density of smooth functions in Sobolev-Orlicz spaces. Zap. Nauč. Semin. POMI 310, 67-81 (2004) translation in J. Math. Sci. (N.S.) 132, 285-294 (2006)

27. Fan, $X L$, Zhao, D: On the spaces $L^{p(x)}(\Omega)$ and $W^{m, p(x)}$. J. Math. Anal. Appl. 263, 424-446 (2001)

28. Kovàcik, O, Rákosník, J: On spaces $L^{p(x)}$ and $W^{k, p(x)}$ Czechoslov. Math J. 41, 592-618 (1991)

\section{Submit your manuscript to a SpringerOpen ${ }^{\circ}$ journal and benefit from:}

- Convenient online submission

- Rigorous peer review

- Open access: articles freely available online

- High visibility within the field

- Retaining the copyright to your article

Submit your next manuscript at $>$ springeropen.com 